\title{
BMJ Open Physical prognostic factors predicting outcome following anterior cruciate ligament reconstruction: protocol for a systematic review
}

\author{
Andrew Middlebrook (D) , ${ }^{1}$ Sheree Bekker (D) , ${ }^{1}$ Nicola Middlebrook (D) , \\ Alison B Rushton (i) ${ }^{2}$
}

To cite: Middlebrook A, Bekker S, Middlebrook N, et al. Physical prognostic factors predicting outcome following anterior cruciate ligament reconstruction: protocol for a systematic review. BMJ Open 2020;10:e033429. doi:10.1136/ bmjopen-2019-033429

- Prepublication history and additional material for this paper are available online. To view these files, please visit the journal online (http://dx.doi org/10.1136/bmjopen-2019033429).

Received 04 August 2019 Revised 01 March 2020 Accepted 09 March 2020

\section{Check for updates}

(c) Author(s) (or their employer(s)) 2020. Re-use permitted under CC BY-NC. No commercial re-use. See rights and permissions. Published by BMJ.

${ }^{1}$ Department of Health, University of Bath, Bath, UK ${ }^{2}$ Centre of Precision Rehabilitation for Spinal Pain, University of Birmingham, Birmingham, UK

Correspondence to Andrew Middlebrook; midd84@hotmail.com

\section{ABSTRACT}

Introduction Injuries of the anterior cruciate ligament (ACL) are a common musculoskeletal complication and can cause significant reduction in patient function and quality of life. Many undergo ACL reconstruction, with high-quality rehabilitation key to successful outcome. Knowledge of physical prognostic factors, such as quadriceps strength, is crucial to inform rehabilitation and has important implications for outcome following $\mathrm{ACL}$ reconstruction. However, these factors predicting outcome are poorly defined. Therefore, the aim of this systematic review is to establish physical prognostic factors predictive of outcome in adults following ACL reconstruction. Outcome will be subdivided into two groups of outcome measures, patient-reported and performance-based. Physical prognostic factors of interest will reflect a range of domains and may be modifiable/non-modifiable. Results will help decide most appropriate management and assist in planning and tailoring preoperative and postoperative rehabilitation. Methods and analysis This systematic review protocol is reported according to the Preferred Reporting Items for Systematic Review and Meta-Analysis Protocols. MEDLINE, CINAHL and EMBASE databases, key journals and grey literature will be searched from inception to July 2019. Prospective cohort studies including participants aged $\geq 16$ years who have undergone ACL reconstruction will be included, with articles focusing on multi-ligament reconstructions and ACL repair surgery, or not published in English excluded. Two independent reviewers will conduct searches, assess study eligibility, extract data, assess risk of bias (Quality in Prognostic Studies tool) and quantify overall quality of evidence (modified Grading of Recommendations, Assessment, Development and Evaluation guidelines). If possible, a meta-analysis will be conducted, otherwise a narrative synthesis will ensue focusing on prognostic factors, risk of bias of included studies and strength of association with outcomes.

Ethics and dissemination Findings will be published in a peer-reviewed journal, presented at conferences and locally to physiotherapy departments. Ethical approval is not required for this systematic review.

PROSPERO registration number CRD42019127732.
Strengths and limitations of this study

- This protocol is written and reported in line with the Preferred Reporting Items for Systematic Review and Meta-Analysis Protocol guidelines.

- This study uses the Quality in Prognostic Studies tool to assess for risk of bias in individual studies, a tool devised specifically for use in prognostic factor review questions.

- This systematic review will use a modified Grading of Recommendations, Assessment, Development and Evaluation method to rate the overall quality of evidence across studies.

- This study will include only prospective cohort studies in the review, which are the gold standard for prognostic research.

- This review will focus on physical prognostic factors only, but it is recognised that other factors can contribute to overall outcome.

\section{INTRODUCTION}

Injuries of the anterior cruciate ligament (ACL) are common ${ }^{1}$ and regarded as one of the most frequently sustained ligamentous injuries within the knee. ${ }^{2}$ Overall populationbased incidence has been documented as 81 per 100000 people, ${ }^{3}$ with approximately 250 000 individuals affected each year in the USA alone, ${ }^{4}$ the majority of whom undergo ACL reconstruction. ${ }^{5}$ With a primary function of reducing and controlling anterior tibial translation, ${ }^{6}$ the ACL is an important ligament in the maintenance of normal knee function, with rupture resulting in a significant musculoskeletal injury. ${ }^{7}$

Preceding and following ACL reconstruction, good-quality rehabilitation programmes or protocols are regarded as key to successful outcome (positive change resulting from ACL reconstruction). ${ }^{8}$ Successful outcome, or research focusing on outcome, is not well defined within the literature, with multiple definitions suggested. ${ }^{9}$ Following 
ACL reconstruction or revision, outcome measures recommended for the evaluation of outcome vary and include both patient-reported outcome measures and performance-based outcome measures. Furthermore, measures which incorporate the domains of the International Classification of Function, Disability and Health (ICF) (body function, activities and participation, body structure and environmental factors) are useful. ${ }^{11}$ Examples of outcome measures used in the literature include return to sport, ${ }^{12}$ improvement in patient-reported outcomes (eg, International Knee Documentation Committee (IKDC) and Knee Injury and Osteoarthritis Outcome Score (KOOS) questionnaires) ${ }^{1314}$ and performance-based outcomes (eg, quadriceps strength). ${ }^{14}$ As highlighted, it is common that a long-term goal is to allow individuals to return to their prior levels of activity. ${ }^{15}$ However, events such as an ACL reconstruction can also have a significant impact on the personal life of the individual, ${ }^{16}$ with prolonged periods of rehabilitation often required. ${ }^{12}$ As a consequence, several recent systematic reviews have attempted to synthesise the evidence relating to the prognostic psychological factors affecting outcome. ${ }^{17-19}$ In addition, several studies $^{13} 20-23$ have suggested that numerous physical prognostic factors can impact and predict the outcome following ACL reconstruction. In the context of this review, we define physical prognostic factors as being physical in nature, including both modifiable factors, for example, quadriceps strength, and non-modifiable factors, for example, age, recognising that both can have a physical effect and influence outcome. However, no recent high-quality systematic review of such physical prognostic factors has been published. Seen as a developing area of research, ${ }^{24}$ prognostic studies and their findings can contribute to clinical decision making and overall patient management. ${ }^{25}$ Given this, it would appear important for both the patient and the team caring for the patient to be aware of potentially important physical prognostic factors, both modifiable and non-modifiable and their respective implications, when considering both preoperative and postoperative expectations, rehabilitation and management.

\section{Status of current literature}

Few systematic reviews have investigated factors predicting successful outcome after ACL reconstruction, ${ }^{26-28}$ where successful outcome could be quantified using the performance-based outcome measures such as return to sport or return to a previous level of activity, or improvement in the patient-reported outcome measure scores. ${ }^{12-14}$ From these reviews, multiple factors have been identified which are associated with poorer outcome (ie, not returning to a previous level of activity or decrease in the outcome measure scores), such as being a smoker, preoperative quadriceps strength deficit of $20 \%$ or more, preoperative knee range of motion limitations (flexion), increased pain, ACL or knee laxity and concomitant meniscal injury. ${ }^{2729}$ Furthermore, factors of re-rupture have been associated with younger age groups and individuals returning to a high level of sport in which pivoting and twisting movements are common. ${ }^{28}$ Predictors of successful outcome include male patients aged $<30$ years with 'normal' body mass index (BMI) and the use of hamstring tendon autografts. ${ }^{26} 27{ }^{29}$ With all reviews, a successful outcome has been associated with improved scores when using a range of outcome measures such as the IKDC and KOOS questionnaires. However, these studies have varied in their inclusion and exclusion criteria around study design and included prognostic factors, with only two reviews assessing risk of bias, ${ }^{27} 28$ making it difficult to draw conclusions.

A recent cohort study by Scherer $e t a l^{20}$ examined factors associated with a more rapid recovery following ACL reconstruction. As a result, the study included a follow-up at 6 months postsurgery and targeted return to a previous level of activity as a primary aim. Conclusions were in agreement with de Valk $e t a l^{26}$ that preoperative factors, such as younger age, 'normal' knee flexion and extension strength and no previous knee surgery, were associated with improved outcome at a 6-month follow-up. However, Scherer $e t a l^{20}$ used a convenience sample from a single clinic, making generalisation of findings difficult.

Further studies have evaluated factors predicting failure following ACL reconstruction, with results showing multiple predictive factors. Both Parkinson $e t a l^{21}$ and Robb et $a l^{22}$ found that meniscal integrity was the strongest predictor of ACL reconstruction failure. However, it should be highlighted that both studies only included single-bundle ACL reconstructions using a hamstring graft and primarily focused on the risk factors for graft failure, as opposed to physical factors predicting outcome. Despite this, these studies were further supported by a large study by Cox $e t a l^{13}$ which found patients who underwent a meniscal repair as well as an ACL reconstruction scored lower on the IKDC and KOOS. Furthermore, other factors such as female gender, ${ }^{23}$ increased BMI, lower levels of education, smoking status and revision of ACL surgery have been found predictive of poorer outcomes, ${ }^{13}$ but the potential for reporting and publication bias in original studies is acknowledged. ${ }^{23}$

\section{Rationale for further research}

These highlighted studies emphasise that there are numerous and wide-ranging potential physical prognostic factors that may predict outcomes following ACL reconstruction and that they can be grouped into either modifiable (eg, quadriceps strength) or non-modifiable factors (eg, age). Despite the continued research into the prognostic factors predicting outcome following ACL reconstruction over the 7 years since the systematic review by de Valk et al, ${ }^{26}$ no rigorous systematic review that utilises and follows the Preferred Reporting Items for Systematic Reviews and Meta-Analyses (PRISMA) guidelines exists. Although a recent systematic review of patient-reported outcome following ACL reconstruction was published by Hamrin Senorski et $a l^{27}$ this only includes publications 
and data from the Scandinavian knee ligament registers and, given the sample of convenience, it cannot be assumed to represent the wider population in countries outside of this region. There is, therefore, a need for a high-quality systematic review to evaluate and synthesise the current body of literature within this important area of research.

As demonstrated by the systematic reviews and studies presented, ${ }^{12-14}$ 26-28 it is apparent that definitions of outcome and outcome measures utilised to determine outcome are wide ranging, with both patient-reported outcome measures and performance-based outcome measures adopted. This highlights a lack of standardisation within the literature ${ }^{30}$ and results in the need for a broad approach to outcome and outcome measures within this review, in order to capture all relevant literature and avoid risk of bias.

\section{Objective}

To establish the physical prognostic factors predictive of outcome in adults following ACL reconstruction. This will, in turn, help clinicians and patients in making decisions on the most appropriate management and assist, where decided appropriate, in planning and tailoring both preoperative and postoperative rehabilitation following ACL reconstruction.

\section{METHODS \\ Design}

A systematic review designed as a result of scoping literature searches, reported in accordance with Preferred Reporting Items for Systematic Review and Meta-Analysis Protocols guidelines (PRISMA-P) ${ }^{31}$ and Cochrane Handbook. $^{32}$

\section{Eligibility criteria}

\section{Inclusion criteria}

\section{Population}

Any patient aged $\geq 16$ years undergoing an ACL reconstruction or revision. If a study includes patients aged both $<16$ years and $\geq 16$ years, the study will be included if it is possible to separate the data so as to only include the participants aged $\geq 16$ years. If this is not possible, the study will be excluded.

\section{Physical prognostic factors}

Based on the previous literature, ${ }^{13} 222326$ 33-35 examples of potential factors to be included are documented in box 1 . However, any additional investigated physical prognostic factors identified in the search will be included. For the purpose of this review, we define physical prognostic factors as being physical in nature, including both modifiable, for example, quadriceps strength, and nonmodifiable, for example, age, recognising that both can have a physical effect and influence outcome.

\section{Study design}

Prospective cohort studies (gold standard design for prognostic reviews) that focus upon prognostic factors

\section{Box 1 Potential physical factors identified}

\author{
Potential physical prognostic factors \\ - Quadriceps strength. \\ - Knee range of movement. \\ - Concomitant meniscal injuries. \\ - Concomitant articular cartilage injuries. \\ - Level of preoperative pain. \\ - Amount of preoperative ACL laxity. \\ - Preinjury activity levels. \\ - History of previous knee surgery. \\ - ACL rupture location (mid-substance). \\ - Graft type-allograft vs autograft. \\ - Graft site-hamstring vs patella tendon. \\ - BMI. \\ - Smoking status (physical effects of smoking). \\ - Time from injury to surgery. \\ - Age. \\ - Gender.
}

$\mathrm{ACL}$, anterior cruciate ligament; BMI, body mass index.

and that include a follow-up, of any time period, will be included within this review. This will include both preoperative and postoperative variables. There will be no restriction on the date of publication.

\section{Timing and setting}

There will be no restriction on length of follow-up and all time points used within included studies will be included. No restriction on setting will be used.

\section{Outcomes}

Given the broad spectrum of outcome measures used to evaluate outcome following surgery within studies, all outcome measures recommended in the evaluation of ACL reconstruction or revision will be included. This can include patient-reported outcome measures or performance-based measures. For the purpose of this review, outcome measures will be classified into domains of outcomes in keeping with the four domains of the ICF. ${ }^{10}$ This allows a more holistic approach in assessment of 'outcome' following ACL reconstruction rather than focusing on one domain or one outcome measure. Outcome measures will include: functional tests, strength, return to previous level of activity, radiological measures, graft failure and complications, and patient-reported outcome measures such as the KOOS and IKDC. Owing to this heterogeneity of outcome measures, narrowing to a specific outcome measure is not possible as it would exclude large bodies of relevant literature. Therefore, any outcome of interest will be included.

\section{Exclusion criteria}

Any study which includes multi-ligament reconstruction of the knee will be excluded from this review. This is due to multi-ligament injuries being more complex and potentially limb threatening, with neurovascular compromise not uncommon, as well as a lack of evidence 
and consensus regarding their optimal management. ${ }^{36}$ Furthermore, due to the differing techniques and elevated risk of re-rupture in ACL repairs compared with ACL reconstruction, ${ }^{37-39}$ any study which evaluates repair surgery will be excluded. Any study not published in English will be excluded.

\section{Information sources}

Comprehensive searches will be performed from inception to July 2019 via the following electronic databases: MEDLINE, CINAHL, EMBASE. Additionally, grey literature searches including the British National Bibliography, OpenGrey and Zetoc will be conducted. Hand searching of key journals (The American Journal of Sports Medicine, The British Journal of Sports Medicine and Knee Surgery, Sports Traumatology and Arthroscopy) will be conducted as well as screening reference lists of included articles which meet the eligibility criteria.

\section{Search strategy}

A search strategy has been developed by modifying a strategy previously utilised within prognostic factor research $^{40}$ and using key terms for the population of interest (ACL reconstruction) together with prognostic search terms. A MEDLINE search strategy will be piloted using relevant MeSH terms where applicable and subsequently developed for other databases. A validated methodological search filter for MEDLINE to identify prognostic studies will be used ${ }^{41}$ and adapted for other databases. No specific restrictions on language or date will be used. The exact MEDLINE search strategy used is provided in online supplementary file 1 .

\section{Data management}

Results from searches will be stored on Endnote V.X8 (Clarivate Analytics) software programme. In doing this, duplicates will be established and eliminated.

\section{Study selection}

Titles and abstracts will be screened against the predetermined inclusion and exclusion criteria and then categorised into include, exclude or undecided. This process will be done independently by two reviewers (AM and $\mathrm{NM}$ ). Where uncertainty exists regarding the eligibility of a paper, the full text will be accessed for clarification. Any disagreement between the two reviewers regarding eligibility of a study will result in discussion between them. The agreement between the two reviewers at each stage will be assessed using Cohen's kappa. ${ }^{42}$ If no agreement is reached, the opinion of an independent third reviewer (ABR) will be obtained. Following title and abstract screening and once the full text articles are obtained, the above screening process will be repeated. The reasons for ineligibility will be documented and a PRISMA flow diagram will be included within the review.

\section{Data extraction process}

A standardised form has been developed based on the information deemed relevant as a result of initial subject searches and utilised to extract information from included studies. This will be done independently by both reviewers, with the lead reviewer then collating the information. A pilot of the data extraction form will initially be performed using five articles, to ensure that all appropriate data is extracted. Following this and if required, the form will be amended before the final data extraction.

\section{Data items}

Data to be extracted from included studies is summarised in table 1 . Should there be any missing data, authors will be contacted by email for further clarification. In the event of more than one article being identified from the same study, authors will be contacted for further information in order to avoid duplication.

\section{Outcomes and prioritisation}

Outcome was identified as any recommended evaluation of ACL reconstruction and revision which could include outcome measures reflecting any domain of the ICF, specifically body function, activities and participation, body structure and environmental factors. Outcome measures may be both patient-reported outcome measures and performance-based outcome measures. Outcome measures used following ACL reconstruction and reflected in an earlier systematic review by de Valk et $a l,{ }^{26}$ include the IKDC, KOOS, Tegner Activity Scale, Lysholm Knee Scoring Scale and the Cincinnati Knee Rating System. It is appreciated that outcomes and follow-up assessment timepoints will be of differing lengths. Short-term ( $<3$ months), medium-term ( $>3$ months, $<12$ months) and long-term ( $>12$ months) outcome will be presented, with the main area of interest being long-term outcome. Long-term outcome will be subdivided to $>12$ months but $<5$ years and $>5$ years in order to capture the potential variabilities in longer-term outcome. The scoping search had identified that it was not possible to predefine one outcome of interest and one timepoint.

\section{Risk of bias in individual studies}

The Quality in Prognostic Studies (QUIPS) tool will be adopted to assess the risk of bias of included studies. The QUIPS tool was created for prognostic factor review questions $^{43}$ and is recommended by the Cochrane Group ${ }^{44}$ to assess the risk of bias in prognostic factor studies and, as such, has been utilised in a previous systematic review relating to physical prognostic factors. ${ }^{45}$ It encompasses six categories-domains of potential biases: study participation, study attrition, prognostic factor measurement, outcome measurement, study confounding, statistical analysis and reporting, and has demonstrated acceptable inter-rater reliability. ${ }^{46}$ Mirroring the selection process, all included studies will be assessed by two independent reviewers and scored for the risk of bias. Any disagreement between the two reviewers will result in discussion between them. The agreement between 
Table 1 Summary of data items to be extracted from included studies

\begin{tabular}{ll}
\hline Content & Data items \\
\hline General study information & Title, authors names, publication date. \\
Study characteristics & Sample size, study design, duration of follow-up, country. \\
\hline Patient characteristics & Age, gender. \\
\hline Type of intervention/ACL reconstruction & Graft site choice, type of graft. \\
Physical prognostic factors & $\begin{array}{l}\text { Examples include but not limited to: quadriceps strength, knee range of } \\
\text { motion, concomitant meniscal injuries, concomitant articular cartilage }\end{array}$ \\
& $\begin{array}{l}\text { injuries, level of preoperative pain, degree of preoperative ACL laxity, } \\
\text { preinjury activity levels, history of previous knee surgery, ACL rupture } \\
\text { location, ACL graft type, ACL graft site, BMI, smoking status, time from } \\
\text { injury to surgery, age and gender. }\end{array}$ \\
\hline $\begin{array}{l}\text { Outcome (encompassing patient-reported outcome } \\
\text { measures / performance-based outcome measures) }\end{array}$ & $\begin{array}{l}\text { Patient-reported outcome measures for example, IKDC, KOOS, Tegner } \\
\text { Activity Scale, Lysholm Knee Scoring Scale and the Cincinnati Knee } \\
\text { Rating System. } \\
\text { Performance-based outcome measures for example, return to sport } \\
\text { and/or a previous level of activity, hop tests, quadricep strength. }\end{array}$ \\
\hline Results & $\begin{array}{l}\text { Main findings, statistical analysis methods. } \\
\text { Other information deemed relevant }\end{array}$ \\
\hline
\end{tabular}

$\mathrm{ACL}$, anterior cruciate ligament; BMI, body mass index; IKDC, International Knee Documentation Committee; KOOS, Knee Injury and Osteoarthritis Outcome Score.

the two reviewers at each stage will be assessed using Cohen's kappa coefficient. ${ }^{42}$ If no agreement is reached, the opinion of an independent third reviewer will be obtained.

\section{Data synthesis}

Subject to homogeneity (consistency of factors across studies) of predictive factors, outcomes and timing of outcome, a meta-analysis will be conducted. This will be dependent on finding three or more studies with adequately homogenous sub-groups. The assessment of clinical, methodological and statistical heterogeneity will take place. The $\mathrm{I}^{2}$ statistic will be used to assess statistical heterogeneity, while methodological heterogeneity will be based on study biases and clinical heterogeneity based on measures of population and prognostic factors. ${ }^{32}$ Studies will be included or excluded for meta-analysis by using the QUIPS tool, whereby high quality, low risk of bias studies are included and high risk of bias studies excluded, with a random effects model being utilised for meta-analysis. If a meta-analysis is not possible, a qualitative best evidence narrative synthesis of results will take place. The narrative synthesis would be focused on the prognostic factors, the risk of bias assessment of included studies, and the strength of association with the outcome. ${ }^{45}$ From scoping searches that have been conducted, cohorts can include revisions as well as primary ACL reconstruction. To be inclusive of all potential factors which can influence outcome, revisions and mixed cohorts will be included in this review. In the event of studies which have focused on revisions only, these studies will be synthesised and evaluated separately.

\section{Meta-biases}

To examine and establish whether any reporting bias exists, thorough searches for unpublished studies will be undertaken. This will involve accessing past conference proceedings of the last 10 years, as well as detailed internet searches. Consistency between protocols and published studies (where protocols are available) will be scrutinised. Given the lack of a trial registry specific to prognostic studies, trial registries will not be searched.

\section{Confidence in cumulative evidence}

Overall quality and strength of the evidence will be examined using the Grading of Recommendations Assessment, Development and Evaluation (GRADE) method. ${ }^{47}$ GRADE is a tool consisting of five components: study design and its limitations, inconsistency of results across studies, indirectness of evidence, imprecision and publication bias designed for interventional reviews. ${ }^{47}$ GRADE has been adapted to be better suited for prognostic factor research ${ }^{48}$ which includes six factors that may decrease quality (phase of investigation, study design and its limitations, inconsistency of results across studies, indirectness of evidence, imprecision and publication bias) and two factors that may increase the quality (moderate or large effect size and exposure-response gradient). ${ }^{48}$

\section{Patient and public involvement}

The focus of this systematic review was developed as a result of working with and rehabilitating many patients following ACL reconstruction surgery and witnessing a broad scale of outcomes along this process. Patients will not be involved in the data collection and analysis of the review. 


\section{Clinical implications of this study}

The outcome of this work will be a summary of the evidence of physical prognostic factors predicting outcome following ACL reconstruction, which will include both modifiable and non-modifiable factors and their implications on recovery. This will build on previous systematic reviews within the area and provide the most recent findings relating to this topic in a high-quality review. This will give both patients and clinicians valuable insight and knowledge to help with decision making relating to patient care and will assist with the management of expectations of all involved. Furthermore, the review will provide vital information which clinicians can utilise to tailor and maximise both preoperative and postoperative rehabilitation programmes with the aim of minimising risk of poor outcome and optimising patient potential following ACL reconstruction.

\section{ETHICS AND DISSEMINATION}

Findings will be published in a relevant international peer-reviewed journal and presented at conferences and more locally to physiotherapy teams and departments.

Twitter Andrew Middlebrook @andymiddlebrook, Sheree Bekker @shereebekker, Nicola Middlebrook @nic_middlebrook and Alison B Rushton @abrushton

Contributors The focus of the systematic review was formulated by $A M, A B R$ and NM. AM is an MSc. student, ABR (lead and external supervisor) and SB (internal supervisor) are supervisors, NM is the second reviewer. AM drafted the initial version of the protocol manuscript, based on guidance and feedback from $A B R, S B$ and NM relating to topic, methodology and analyses. ABR reviewed and commented on all drafts of the protocol. All authors have approved the final manuscript.

Funding The authors have not declared a specific grant for this research from any funding agency in the public, commercial or not-for-profit sectors.

Competing interests None declared.

Patient consent for publication Not required.

Provenance and peer review Not commissioned; externally peer reviewed.

Open access This is an open access article distributed in accordance with the Creative Commons Attribution Non Commercial (CC BY-NC 4.0) license, which permits others to distribute, remix, adapt, build upon this work non-commercially, and license their derivative works on different terms, provided the original work is properly cited, appropriate credit is given, any changes made indicated, and the use is non-commercial. See: http://creativecommons.org/licenses/by-nc/4.0/.

\section{ORCID iDs}

Andrew Middlebrook http://orcid.org/0000-0002-2254-0783

Sheree Bekker http://orcid.org/0000-0003-0161-6280

Nicola Middlebrook http://orcid.org/0000-0003-2154-5723

Alison B Rushton http://orcid.org/0000-0001-8114-7669

\section{REFERENCES}

1 Meuffels DE, Favejee MM, Vissers MM, et al. Ten year follow-up study comparing conservative versus operative treatment of anterior cruciate ligament ruptures. A matched-pair analysis of high level athletes. Br J Sports Med 2009;43:347-51.

2 Duncan KJ, Chopp-Hurley JN, Maly MR. A systematic review to evaluate exercise for anterior cruciate ligament injuries: does this approach reduce the incidence of knee osteoarthritis? Open Access Rheumatol 2016;8:1-16.

3 Frobell RB, Lohmander LS, Roos HP. Acute rotational trauma to the knee: poor agreement between clinical assessment and magnetic resonance imaging findings. Scand J Med Sci Sports 2007;17:109-14.
4 Griffin LY, Albohm MJ, Arendt EA, et al. Understanding and preventing noncontact anterior cruciate ligament injuries: a review of the HUNT Valley II meeting, January 2005. Am J Sports Med 2006;34:1512-32.

5 Paterno MV. Non-Operative care of the patient with an ACL-Deficient knee. Curr Rev Musculoskelet Med 2017;10:322-7.

6 Smith TO, Postle K, Penny F, et al. Is reconstruction the best management strategy for anterior cruciate ligament rupture? A systematic review and meta-analysis comparing anterior cruciate ligament reconstruction versus non-operative treatment. Knee 2014;21:462-70.

7 Tsoukas D, Fotopoulos V, Basdekis G, et al. No difference in osteoarthritis after surgical and non-surgical treatment of ACLinjured knees after 10 years. Knee Surg Sports Traumatol Arthrosc 2016;24:2953-9.

8 Lynch AD, Logerstedt DS, Grindem H, et al. Consensus criteria for defining 'successful outcome' after ACL injury and reconstruction: a Delaware-Oslo ACL cohort investigation. Br J Sports Med 2015;49:335-42.

9 Jefford M, Stockler MR, Tattersall MHN. Outcomes research: what is it and why does it matter? Intern Med J 2003;33:110-8.

10 WHO. International classification of functioning, disability and health. Geneva, Switzerland: World Health Organisation, 2001.

11 Escorpizo R, Bemis-Dougherty A. Introduction to special issue: a review of the International classification of functioning, disability and health and physical therapy over the years. Physiother Res Int 2015;20:200-9.

12 Ardern CL, Webster KE, Taylor NF, et al. Return to the preinjury level of competitive sport after anterior cruciate ligament reconstruction surgery: two-thirds of patients have not returned by 12 months after surgery. Am J Sports Med 2011;39:538-43.

13 Cox CL, Huston LJ, Dunn WR, et al. Are articular cartilage lesions and meniscus tears predictive of IKDC, KOOS, and Marx activity level outcomes after anterior cruciate ligament reconstruction? A 6-year multicenter cohort study. Am J Sports Med 2014;42:1058-67.

14 Pietrosimone BG, Lepley AS, Ericksen HM, et al. Quadriceps strength and corticospinal excitability as predictors of disability after anterior cruciate ligament reconstruction. J Sport Rehabil 2013;22:1-6.

15 Ahn JH, Lee SH. Risk factors for knee instability after anterior cruciate ligament reconstruction. Knee Surg Sports Traumatol Arthrosc 2016;24:2936-42.

16 Eggerding V, Meuffels DE, Bierma-Zeinstra SMA, et al. Factors related to the need for surgical reconstruction after anterior cruciate ligament rupture: a systematic review of the literature. J Orthop Sports Phys Ther 2015;45:37-44.

17 Everhart JS, Best TM, Flanigan DC. Psychological predictors of anterior cruciate ligament reconstruction outcomes: a systematic review. Knee Surg Sports Traumatol Arthrosc 2015;23:752-62.

18 Ross CA, Clifford A, Louw QA. Intrinsic factors associated with return to sport after anterior cruciate ligament reconstruction: a systematic review. S Afr J Physiother 2015;71:230-30.

19 Coronado RA, Bird ML, Van Hoy EE, et al. Do psychosocial interventions improve rehabilitation outcomes after anterior cruciate ligament reconstruction? A systematic review. Clin Rehabil 2018;32:287-98.

20 Scherer JE, Moen MH, Weir A, et al. Factors associated with a more rapid recovery after anterior cruciate ligament reconstruction using multivariate analysis. Knee 2016;23:121-6.

21 Parkinson B, Robb C, Thomas M, et al. Factors that predict failure in anatomic Single-Bundle anterior cruciate ligament reconstruction. Am J Sports Med 2017;45:1529-36.

22 Robb C, Kempshall P, Getgood A, et al. Meniscal integrity predicts laxity of anterior cruciate ligament reconstruction. Knee Surg Sports Traumatol Arthrosc 2015;23:3683-90.

23 Tan SHS, Lau BPH, Khin LW, et al. The importance of patient sex in the outcomes of anterior cruciate ligament reconstructions: a systematic review and meta-analysis. Am J Sports Med 2016;44:242-54.

24 Steyerberg EW, Moons KGM, van der Windt DA, et al. Prognosis research strategy (progress) 3: prognostic model research. PLoS Med 2013;10:e1001381.

25 Hemingway H, Croft P, Perel P, et al. Prognosis research strategy (progress) 1: a framework for researching clinical outcomes. BMJ 2013;346:e5595.

26 de Valk EJ, Moen MH, Winters M, et al. Preoperative patient and injury factors of successful rehabilitation after anterior cruciate ligament reconstruction with single-bundle techniques. Arthroscopy 2013;29:1879-95.

27 Hamrin Senorski E, Svantesson E, Baldari A, et al. Factors that affect patient reported outcome after anterior cruciate ligament 
reconstruction-a systematic review of the Scandinavian knee ligament registers. Br J Sports Med 2019;53:410-7.

28 Wiggins AJ, Grandhi RK, Schneider DK, et al. Risk of secondary injury in younger athletes after anterior cruciate ligament reconstruction: a systematic review and meta-analysis. Am J Sports Med 2016;44:1861-76.

29 van Melick N, van Cingel REH, Brooijmans F, et al. EvidenceBased clinical practice update: practice guidelines for anterior cruciate ligament rehabilitation based on a systematic review and multidisciplinary consensus. Br J Sports Med 2016;50:1506-15.

30 Stucki G, Reinhardt JD, Grimby G, et al. Developing "Human Functioning and Rehabilitation Research" from the comprehensive perspective. J Rehabil Med 2007;39:665-71.

31 Moher D, Shamseer L, Clarke M, et al. Preferred reporting items for systematic review and meta-analysis protocols (PRISMA-P) 2015 statement. Syst Rev 2015;4:1.

32 Higgins J, Green S. Cochrane Handbook for systematic reviews of interventions, 2011. Available: http://handbook-5-1.cochrane.org [Accessed 28 Apr 2019].

33 Swirtun LR, Renström P. Factors affecting outcome after anterior cruciate ligament injury: a prospective study with a six-year followup. Scand J Med Sci Sports 2008;18:318-24.

34 Spindler KP, Huston LJ, Wright RW, et al. The prognosis and predictors of sports function and activity at minimum 6 years after anterior cruciate ligament reconstruction: a population cohort study. Am J Sports Med 2011;39:348-59.

35 Krismer AM, Gousopoulos L, Kohl S, et al. Factors influencing the success of anterior cruciate ligament repair with dynamic intraligamentary stabilisation. Knee Surg Sports Traumatol Arthrosc 2017:25:3923-8.

36 Levy BA, Dajani KA, Whelan DB, et al. Decision making in the multiligament-injured knee: an evidence-based systematic review. Arthroscopy 2009;25:430-8.
37 Feagin JA, Curl WW. Isolated tear of the anterior cruciate ligament: 5-year follow-up study. Am J Sports Med 1976;4:95-100.

38 Marshall JL, Warren RF, Wickiewicz TL, et al. The anterior cruciate ligament: a technique of repair and reconstruction. Clin Orthop Relat Res 1979;143:97-106.

39 Zysk SP, Refior HJ. Operative or conservative treatment of the acutely torn anterior cruciate ligament in middle-aged patients. A follow-up study of 133 patients between the ages of 40 and 59 years. Arch Orthop Trauma Surg 2000;120:59-64.

40 Rushton A, Heneghan N, Heijmans MW, et al. Natural course of pain and disability following primary lumbar discectomy: protocol for a systematic review and meta-analysis. BMJ Open 2016;6:e010571.

41 Wilczynski NL, Haynes RB, tH T. Developing optimal search strategies for detecting clinically sound prognostic studies in MEDLINE: an analytic survey. BMC Med 2004;2:23.

42 Landis JR, Koch GG. The measurement of observer agreement for categorical data. Biometrics 1977;33:159-74.

43 Hayden JA, Côté P, Bombardier C. Evaluation of the quality of prognosis studies in systematic reviews. Ann Intern Med 2006; $144: 427-37$.

44 Cochrane group, 2019. Available: https://methods.cochrane.org/ prognosis/our-publications [Accessed 28 Apr 2019].

45 Rushton A, Zoulas K, Powell A, et al. Physical prognostic factors predicting outcome following lumbar discectomy surgery: systematic review and narrative synthesis. BMC Musculoskelet Disord 2018;19:326.

46 Hayden JA, van der Windt DA, Cartwright JL, et al. Assessing bias in studies of prognostic factors. Ann Intern Med 2013;158:280-6.

47 Guyatt GH, Oxman AD, Schünemann HJ, et al. Grade guidelines: a new series of articles in the Journal of clinical epidemiology. $J$ Clin Epidemiol 2011:64:380-2.

48 Huguet A, Hayden JA, Stinson J, et al. Judging the quality of evidence in reviews of prognostic factor research: adapting the grade framework. Syst Rev 2013;2:71. 